\title{
Evaluation of microRNA-223 and microRNA-125a expression association with STAT3 and Bcl2 genes in blood leukocytes of CLL patients: a case-control study
}

\author{
Nader Davari ${ }^{1}$, Fatemeh Ahmadpour ${ }^{2}$, Ali Asghar Kiani ${ }^{3,4}$, Mozhgan Azadpour ${ }^{5}$ and Zari Tahannejad Asadi ${ }^{1,6^{*}}$ (1)
}

\begin{abstract}
Objective: In chronic lymphocytic leukemia (CLL), lack of expression or dysregulation of some special miRs disrupts apoptosis of malignant cells; thereby miR expression can enhance cell proliferation, disease progression and decrease patient survival.

Results: $30 \mathrm{CLL}$ patients and 20 healthy individuals participated in the study. RNA was extracted to evaluate the expression of miR-125, miR-223, BCL-2 and signal transducer and transcription 3 activator (STAT3) genes; quantitative Real Time- PCR (Q-RT-PCR) was performed. MiR-125a and miR-223 expression decreased in the patients compared to the control group (P-Value:0.001). BCL-2 and STAT3 which are the target genes of these two miRs, showed increased expression, in the patients compared to the control subjects (P-Value: 0.001 and P-Value: 0.64 respectively). A significant reverse relationship was found between miR-125a and BCl-2 expression and WBC count. Significantly, miR-223 expression was associated with smoking in patients (P-Value: 0.007 ). Also, these miRs may have regulatory effects by controlling white blood cell (WBC) production based on the inverse correlation with WBC count and hemoglobin $(\mathrm{Hb})$ concentration. Finally, miR-223 can be used as a prognostic factor in CLL patients; miR-125a may be useful for evaluating the therapeutic approaches based on the inverse link with $\mathrm{BCl}-2$.
\end{abstract}

Keywords: Chronic lymphocytic leukemia, MicroRNA-223, MicroRNA-125a, BCL2, STAT3, Prognostic

\section{Introduction}

Chronic lymphocytic leukemia (CLL) is a type of malignancy which is characterized by accumulation of lymphocytes (mostly B lymphocytes) in bone marrow (BM), lymph nodes, and peripheral blood (PB) [1]. In fact, it is a development and maturation disorder of lymphocytes. CLL is the most common adult malignancy in the western societies [2]; the average age of patients is 65 years and the incidence is twice as high in men $[2,3]$. The main

\footnotetext{
*Correspondence: Aztahannjad@yahoo.com

${ }^{1}$ Thalassemia \& Hemoglobinopathy Research Center, Health Research Institute, Ahvaz Jundishapur University of Medical Sciences, Ahvaz, Iran Full list of author information is available at the end of the article
}

etiology of CLL has not been identified so far, and many factors and pathways had been investigated to describe the CLL pathogenesis [4].

Recent studies have shown that MicroRNAs (miRs) can be used as therapeutic tools for CLL patients; they play regulatory roles in gene transcription and expression $[5,6]$. In fact, miRs are series of non-coding RNAs, consist of 20-22 nucleotides; the two major classes of target genes of miRs are tumor suppressors and oncogenes. In CLL, usually miRs which target tumor suppressor genes are impaired, while the function of miRs that target oncogenes is increased [7, 8]. MiR-125a has been recognized as a tumor suppressor in many cancers; it is effective in malignancies pathogenesis such 
as breast cancer by affecting many transcription factors such as STAT3 and ERBB2 $[9,10]$. MiR-223 is another tumor suppressor which targets the STAT3 factor, similar to miR-125 [11, 12].

In this study, we aimed to evaluate miR-125a and miR-223 expression in CLL and control subjects; in the following we analyzed miRs expression relationship with the STAT3 and BCL2 genes expression, as well as their clinical symptoms.

\section{Main text}

\section{Materials and methods}

Study design: CLL patients were diagnosed based on BM aspiration, cytogenetic study, immunophenotype, and morphological findings. The patients $(n=30)$ under study were selected among those who referred to Ahvaz Baqaei hospital during 2018-2019. Inclusion criteria included CLL patients who had complete and accessible clinical information, without any underlying diseases; the malignancy was confirmed by two expert oncologists. Patients with underlying disease and who did not have complete clinical documents were excluded from the research. Also, 20 age matched control personals were entered the study.

$10 \mathrm{ml}$ of $\mathrm{PB}$ was collected from each individual in EDTA anticoagulant tubes. All demographic data of studied individuals are listed in Table1. 60\% of CLL patients had agricultural related occupations, $20 \%$ were employees and $16.7 \%$ were housewives; $3.3 \%$ of them were freelancers.

This study was approved by the local ethics committee of Ahvaz Jundishapur University of Medical Sciences (AJUMS.REC.1397.763); the informed consent was completed by whole of subjects.

Table 1 Demographical information of patients and controls

\begin{tabular}{lllc}
\hline Variables & \multicolumn{2}{l}{ Mean \pm SD } & P-value \\
\cline { 2 - 3 } & Patient $(\mathbf{n}=\mathbf{3 0})$ & Control $(\mathbf{n}=\mathbf{2 0})$ & \\
\hline Age(year) & $63.23 \pm 6.35$ & $54.93 \pm 5.53$ & 0.51 \\
Sex(Female/Male) & $11 / 19$ & $10 / 10$ & 0.26 \\
Height & $168.86 \pm 17.17$ & $169.93 \pm 8.75$ & 0.67 \\
Weight & $78.64 \pm 33$ & $73.18 \pm 17.88$ & 0.90 \\
BMI $\left(\mathrm{kg} / \mathrm{m}^{2}\right)$ & $28.54 \pm 14.23$ & $25.08 \pm 4.39$ & 0.87 \\
Hb $\left(\mathrm{g} / \mathrm{dl}^{2}\right)$ & $10.46 \pm 1.91$ & $14.10 \pm 1.01$ & 0.08 \\
WBC $\left(10^{3} / \mathrm{mm}^{3}\right)$ & $52.28 \pm 66.99$ & $7.06 \pm 1.18$ & $<0.0001$ \\
PLT $\left(10^{3} / \mathrm{mm}^{3}\right)$ & $105.76 \pm 57.61$ & $224.31 \pm 47.99$ & $<0.0001$ \\
\hline
\end{tabular}

\section{miRNA expression analysis}

After sample collection, PB mononuclear cells were isolated by Ficoll-Hypaque density gradient centrifugation method; mRNA isolation was performed according to the Biomaxell kit manufacturer protocol. The extracted RNA was evaluated by light absorption at $260 \mathrm{~nm}$; in the next step cDNA synthesis was performed according to the yektatajhiz azma kit protocol. cDNA synthesis temperature cycle conditions have been described as following, initial denaturation for $5 \mathrm{~min}$ at $70^{\circ} \mathrm{C}, 1 \mathrm{~h}$ at $42^{\circ} \mathrm{C}$, and finally 5 min at $70{ }^{\circ} \mathrm{C}$.

List of the used materials for cDNA synthesis is as follows, $1000 \mathrm{ng}$ total RNA, $1 \mu \mathrm{L}$ Oligo dt primer, $4 \mu \mathrm{L}$ first strand buffer, $1 \mu \mathrm{L}$ dNTP, $1 \mu \mathrm{L}$ M-Mlv, $0.5 \mu \mathrm{L}$ Rnase inhibitor and $20 \mu \mathrm{L}$ Diethyl pyrocarbonate (DEPC) water.

Dedicated primers were used for stem loop miR-125a, stem loop miR-223 and stem loop U6, instead of oligo dt primer for cDNA synthesis of microRNAs. The cited stem loops sequences are as follows.

Stem Loop miR-125a-5P: 5'-GTCGTATCCAGT GCAGGG TCC GAG GTA T TC GCA CTG GATACG ACtcacag- $3^{\prime}$.

Stem Loop miR-223-3P: 5'-GTCGTATCCAGT GCAGGG TCC GAG GTA T TC GCA CTG GATACG ACtggggt- $3^{\prime}$.

Stem Loop U6: 5'-GTCGTATCCAGTGCAGGGTCC GAGGTATTCGCACTGGA TACGACaaaata- $3^{\prime}$.

The temperature cycle for cDNA synthesis included initial denaturation for $5 \mathrm{~min}$ at $70^{\circ} \mathrm{C}$ and $1 \mathrm{~h}$ at $37^{\circ} \mathrm{C}$, and 5 min at $70{ }^{\circ} \mathrm{C}$.

Then, Q-RT-PCR was used to evaluate the expression of miR-125, miR-223, BCL-2 and STAT3. Temperature cycle included initial denaturation for $15 \mathrm{~min}$ at $\left.95^{\circ} \mathrm{C},\right) 15 \mathrm{~s}$ at $95^{\circ} \mathrm{C}$ and $1 \mathrm{~min}$ at $60^{\circ} \mathrm{C}$ (with 40 cycles in 20 $\mu \mathrm{L}$ of PCR master mix. It contained $10 \mu \mathrm{L}$ of SYBR-Green QPCR Master Mix (amplicon), o.4 $\mu \mathrm{L}$ forward primer, $0.4 \mu \mathrm{L}$ reverse primer, $1 \mu \mathrm{L}$ cDNA and $8.2 \mu \mathrm{L}$ of RNase free water for GAPDH, BCL-2 and STAT3; in addition, the following materials were added for U6, miR-125 and miR-223, $10 \mu \mathrm{L}$ of SYBR-Green QPCR Master Mix, o.8 $\mu \mathrm{L}$ forward primer, $0.8 \mu \mathrm{L}$ reverse primer, $1 \mu \mathrm{L}$ cDNA and 7.4 $\mu \mathrm{L}$ of RNase free water.

U6 and GAPDH were used as internal controls. mRNA primers were designed by using human Nucleotide BLAST program and based Stem Loop assay for miRs [13]. The sequences of the used primers are listed in Table 2.

\section{Statistical analysis}

Statistical analyses were performed using SPSS 23.0. The data were expressed as mean \pm SD; $P$ values $<0.05$ were considered statistically significant. Gene expression was 
Table 2 Sequence primers used for Q-RT-PCR

\begin{tabular}{|c|c|c|}
\hline & Forward & Reverse \\
\hline miR-125 & 5'-CGGCGCTCCCTGAGACCCTTTA-3' & 5'-GTGCAGGGTCCGAGGT-3' \\
\hline $\operatorname{miR}-223$ & 5'-GCAGAGTGTCAGTTTGTCAAAT-3' & 5'-GTGCAGGGTCCGAGGT-3' \\
\hline U6 & 5'-CTCGCTTCGGCAGCACA-3' & 5'-GTGCAGGGTCCGAGGT-3' \\
\hline $\mathrm{BCL} 2$ & 5'-AGGATAACGGAGGCTGGGATG-3' & 5'-CATATTTGTTTGGGGCATGT-3' \\
\hline STAT3 & 5'-GCTGCCCCATACCTGAAGAC-3' & 5'-GGTGAGGGACTCAAACTGCC-3' \\
\hline GAPDH & 5'-GGTCGGAGTCAACGGATTTGG-3' & 5'-TGATGACAAGCTTCCCGTTCT-3' \\
\hline
\end{tabular}

analyzed by $2^{-\Delta \Delta C T}$ method. Mann-Whitney, Roc curve and spearman tests were applied for analysis of the data.

\section{Result}

Expression of miR-125a, miR-223, BCL2 and STAT3

miR-125a and miR-223 expression was increased in healthy individuals compared to the CLL patients, which was statistically significant (P-Value: 0.001 ). The analysis indicated that STAT3 and BCL2 expression was higher in the CLL patients. There is insufficient evidence to support the claim that STAT3 expression differs meaningfully between the two groups (P-Value: 0.64), while BCL2 expression significantly vary between the case and control group (P-Value: 0.001) (Fig. 1).

\section{Association between miR-125a and miR-223} with demographical information in patients Based on Additional file 1: Table S1, patients who smoked $(n=5)$ significantly express lower level of miR223 (P-Value: 0.001) compared to non-smokers $(n=25)$. However, there was no significant relationships between other parameters and the cited miRs expression.

\section{Correlation between miR-125a and miR-223 with laboratory parameters}

According to the results, the expression level of miR$125 \mathrm{a}$ in healthy group was correlated with BCL2 gene, inversely (P-Value: 0.02 and total population (case and control) P-value: 0.001). On the other hand, there was a significant inverse relationship between white blood cell count (P-Value: 0.01 ) in the patients group. A significant direct correlation was found between miR-125a expression and hemoglobin concentration in the patient and control individuals (P value: 0.003 ). (Additional file 1 : Figure S2). Also, the expression level of miR-223 was inversely correlated with age in the patients group and the whole population ( $\mathrm{P}$ value: $0.014, \mathrm{P}$-Value $<0.001$ respectively).

In the whole population, a significant direct relationship was found between miR-223 expression and hemoglobin concentration (P value: 0.012 ); similar relationship was found between the two miRs expression ( $\mathrm{P}$ value:
0.001). A significant inverse link was observed between miR-223 and white blood cell count (P value 0.002 ) (Additional file 1: Figure S1).

\section{ROC curve analysis}

An effective and well-known method for evaluating a laboratory test (generally a diagnostic procedure) is receiver operating characteristic (ROC); the ROC curve can answer the question of whether a variable can be a suitable biomarker for a disease. This curve is based on sensitivity and specificity; the area under the curve indicates the diagnostic power of the test. According to the Additional file 1: Figures S3 and S4, miR-125a-5p, miR223-3P and BCL2 gene expression are considered as useful biomarkers for CLL progression monitoring ( $\mathrm{p}$ value $<0.001$.

\section{Discussion}

CLL is one of the hematologic malignancy that characterized by accumulation of B-cells in PB and BM [14]. Due to the prevalence of CLL in Western societies, many studies have been done to identify therapeutic and prognostic markers $[15,16]$. MiRs have wide variety of roles in cell structure and metabolism, but they appear to be most important in regulating hematopoiesis and producing different blood cell lines [17, 18]; miRs expression or function impairment is associated with some diseases, including CLL. The expression of some miRs can be associated with malignant cell proliferation inhibition or increased survival of patients [19,20]. Recent studies have shown that some miRs can be used as a prognostic factor, since they target many involved genes in cell cycle and apoptosis [21].

Ahmadvand study evaluated miR-125a as a biomarker in CLL patients; decreased miR-125 expression was reported in the patients [22]. Another study by Rigolin and associates was performed to determine the association between miR-125a expression and genetic abnormalities in CLL patients; they discovered that reduced miR-125a expression is associated with more genetic abnormalities in CLL individuals [23]. As it was mentioned in the previous part, miR-125a expressed in lower 


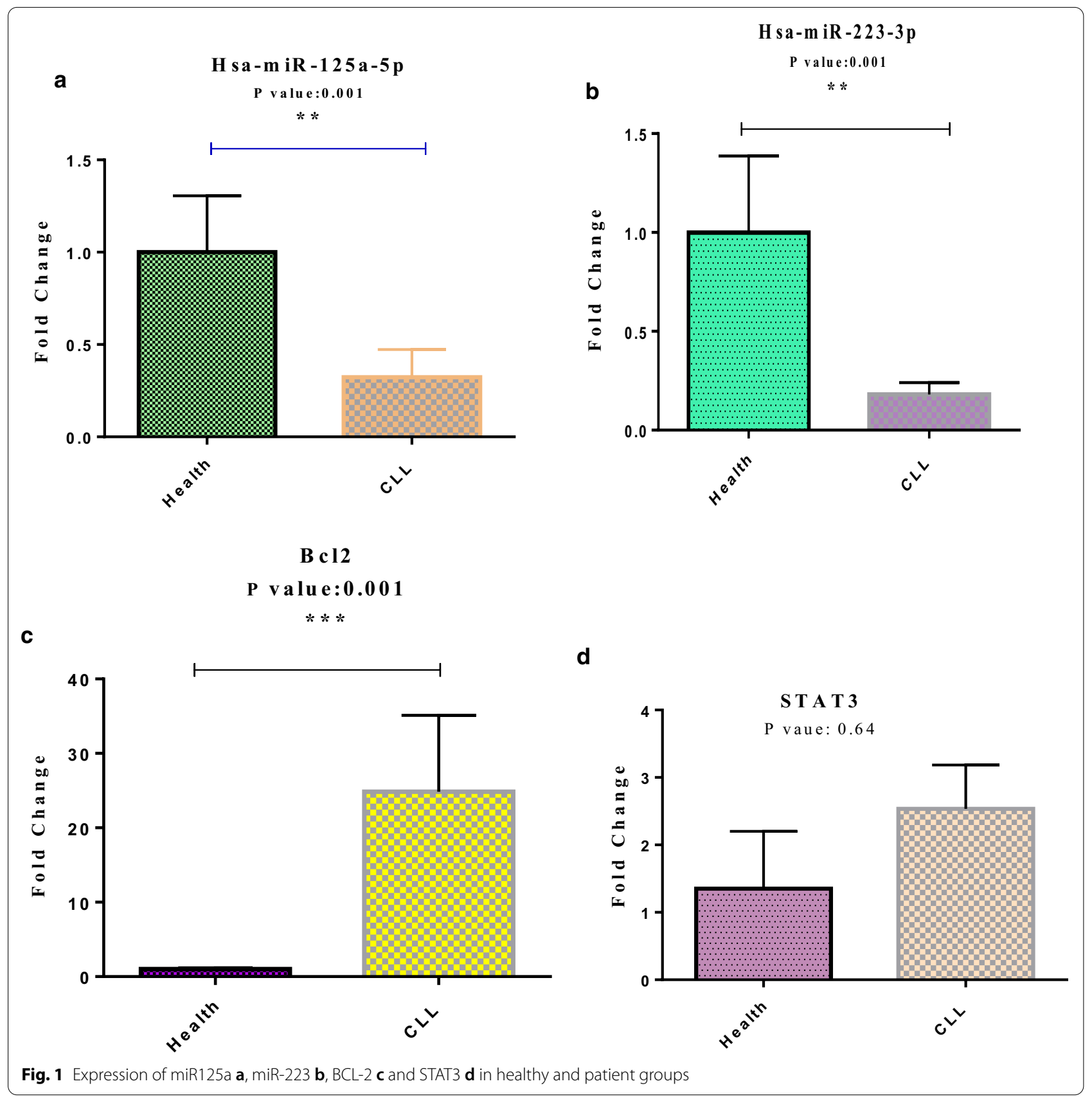

level in the patients under the present study compared to the controls; the reduction was statistically significant (P-Value: 0.001) (Fig. 1a).

A study by Vicente.et al. [24] found that miR-223 expression was reduced in CLL patients lacking the immunoglobulin heavy chain mutation, which was associated with poor prognosis and disease progression. Another study by Zhou confirmed Vicenta's findings; Zhou and coworkers declared that reduced miR-223 expression in CLL patients is associated with aggressive disease and decreased response to treatment [25]. In the present study, miR-223 reduced expression was observed in CLL patients, similar to miR-125a (P-Value: 0.001). It seems that miR-223 is a suitable marker to differentiate between the two groups of cancer and healthy individuals, based on the ROC Curve test for high percent, almost $>80 \%$ (Fig. $1 \mathrm{~b}$ and Additional file 1: Figures S4).

Given that, miR-125a and miR-223 are involved in the pathogenesis and treatment response of patients by 
targeting some genes; evaluating their association with clinical and laboratory parameters may provide useful evidences to improve the importance of these miRs as a prognostic factor. The study by Stamatopoulos.et al. [11] reported a significant relationship between miR-223 and Binet staging system of CLL patients (P-Value: 0.01 ); however, there was no significant relationships between miR-223 and gender (P-Value: 0.49). Toward relation of demographic information and clinical findings of CLL patients, Zhou.et al. did not observe any significant relationships between miR-223, age and leukocyte count (PValue: 0.23 for age and P-Value: 0.21 for leukocytes).

In contrast to age parameter, miR-223 expression was meaningfully related to the patients stage based on the Binet system (P-Value: 0.00) [25]. In the present study none of the cited miRs expression was correlated to the demographic data, except miR-223 which was significantly associated with smoking in CLL patients (P-Value: 0.007) (Additional file 1: Table S1). In fact, these two miRs serve as tumor suppressors which increase therapy response and patient survival; they prevent malignant cells production and induce apoptosis.

Thus, it has been proved that the two BCL-2 and STAT3 genes, which are involved in the proliferation and inhibition of malignant cell apoptosis, are targeted by these two miRs. To this end, Tong.et al. [26] designed a study to investigate the effect of miR-125a on BCL-2 expression in colon cancer cells. They concluded that increased expression of miR-125a is associated with decreased expression of BCL-2, in colon cancer cells. A new finding in our clinical study is invers relation of miR-125a expression to bcl2. It suggests the important role of miR-125a to prevent CLL progression by BCL-2 expression reduction. It could be a therapeutic goal in the future (Additional file 1: Figure S2).

Bozec and associates reported reverse correlation expression of miR-223 and STAT3 in cancer cells; STAT3 is one of the key signaling factors in malignant cell proliferation [27]. According to the results of the present study, BCL-2 and STAT3 expression was higher in the patients than the healthy group; this difference was statistically significant for the BCL-2 as opposed to the STAT3 (P-Value: 0.001 for BCL-2 and P-Value: 0.64 for STAT3) (Fig. 1c, d).

\section{Conclusion}

Finally, given that miR-125a and miR-223 increase in CLL patients and target both BCL-2 and STAT3, it can be concluded that miR-223 expression may be used as a suitable prognostic factor for CLL; miR125a is an appropriate marker for treatment based on the BCL2 link, too.

According to the inverse correlation of the two miRs with WBCs count and $\mathrm{Hb}$ concentration, it can be concluded that these miRs expression may have regulatory effect by controlling WBC proliferation. A significant inverse relationship between miR-223 and smoking suggests a new idea for further researches on smoking, miRs and related molecular signaling.

\section{Limitations}

It is better to monitor the expression of miR-125 and miR-223 during the treatment procedure and evaluate the response to treatment. Evaluation of miR-125 and miR-223 expression in relation to patient survival has not been studied, yet; also, the relationship between these two and expressed CD markers, should be investigated, before and after treatment.

\section{Supplementary Information}

The online version contains supplementary material available at https://doi. org/10.1186/s13104-020-05428-0.

Additional file 1: Table S1. Association between miR125a and 223 with clinico-pathological parameters. Figure S1. Inverse and significant relationship between miR-125a gene expression and white blood cell count in patients. Spearman correlation, $p$ value $\leq 0.05$. Figure S2. Significant inverse relationship between miR-125a $\triangle \mathrm{CT}$ and $\mathrm{BCL} 2 \triangle \mathrm{CT}$ in healthy group. Spearman correlation, $p$ value $\leq 0.01$. Figure $\mathbf{S 3}$. The area under the curve corresponding to the ROC analysis of Has-miR-125a and HasmiR-223 variables in two patient and healthy groups. Figure S4. The area under the curve corresponding to the ROC analysis of BCL2 in two healthy and patient groups.

\section{Acknowledgements}

This work was financially supported by grant TH9718 from the Vice Chancellor for Research Affairs of Ahvaz Jundishapur University of Medical Sciences, Ahvaz, Iran.

\section{Authors' contributions}

ZT has conceived the manuscript and revised it. ND and AK wrote the manuscript. FA and MA provided clinical data and information and performed the technical tests. Also, FA and MA analysis and interpretation of data. All authors read and approved the final manuscript.

\section{Funding}

This work was financially supported by the grant TH9718 from the vice chancellor for research affairs of Ahvaz Jundishapur University of Medical Sciences. This paper is issued from the thesis of Nader Davari. ND and AK wrote the manuscript. FA and MA provided clinical data and information and performed the technical tests. Also, FA and MA analysis and interpretation of data.

\section{Availability of data and materials}

The datasets used analyzed during the current study are available from the corresponding author on reasonable request.

Ethics approval and consent to participate

All the procedures performed in the studies involving human participants were in accordance with ethical standards of local ethics committee of Ahvaz Jundishapur University of Medical Sciences (IR.AJUMS.REC.1397.763), as well as 1964 Helsinki declaration. Written informed consent was obtained from all patients and normal subjects.

Consent for publication

Not applicable. 


\section{Competing interests}

The authors declare that they have no competing interests.

\section{Author details}

${ }^{1}$ Thalassemia \& Hemoglobinopathy Research Center, Health Research Institute, Ahvaz Jundishapur University of Medical Sciences, Ahvaz, Iran. ${ }^{2}$ Department of Clinical Biochemistry, Faculty of Medicine, Ahvaz Jundishapur University of Medical Sciences, Ahvaz, Iran. ${ }^{3}$ Department of Hematology and Blood Transfusion, Lorestan University, Khoramabad, Iran. ${ }^{4}$ Hepatitis Research Center Lorestan University of Medical Sciences, Khorramabad, Iran. ${ }^{5}$ Razi Herbal Medicines Research Center, Lorestan University of Medical Sciences, Khorramabad, Iran. ${ }^{6}$ Department of Laboratory Sciences, Faculty of Paramedicine, Ahvaz Jundishapur University of Medical Sciences, Ahvaz, Iran.

Received: 21 September 2020 Accepted: 22 December 2020 Published online: 11 January 2021

\section{References}

1. Schulz H, Bohlius JF, Trelle S, Skoetz N, Reiser M, Kober T, et al. Immunochemotherapy with rituximab and overall survival in patients with indolent or mantle cell lymphoma: a systematic review and meta-analysis. J Natl Cancer Inst. 2007:99(9):706-14.

2. Rout ED, Burnett RC, Labadie JD, Yoshimoto JA, Avery AC. Preferential use of unmutated immunoglobulin heavy variable region genes in Boxer dogs with chronic lymphocytic leukemia. PLoS ONE. 2018;13(1):e0191205.

3. Nabhan C, Rosen ST. Chronic lymphocytic leukemia: a clinical review. JAMA. 2014;312(21):2265-76.

4. Strati P, Jain N, O'Brien S, editors. Chronic lymphocytic leukemia: diagnosis and treatment. In: Mayo Clinic Proceedings; 2018. Elsevier.

5. Balatti $\mathrm{V}$, Pekarky Y, Croce CM. Role of microRNA in chronic lymphocytic leukemia onset and progression. J Hematol Oncol. 2015;8(1):12.

6. Shahjahani M, Abroun A, Saki N, Bagher Mohammadi SM, Rezaeeyan H. STAT5: from pathogenesis mechanism to therapeutic approach in acute leukemia. Lab Med. 2020;51(4):345-51.

7. Iorio MV, Croce CM. MicroRNA dysregulation in cancer: diagnostics, monitoring and therapeutics. A comprehensive review. EMBO Mol Med. 2012:4(3):143-59.

8. Rezaeeyan H, Hassani SN, Barati M, Shahjahani M, Saki N. PD-1/PD-L1 as a prognostic factor in leukemia. J Hematopathol. 2017:10(1):17-24.

9. Fan Z, Cui H, Xu X, Lin Z, Zhang X, Kang L, et al. MiR-125a suppresses tumor growth, invasion and metastasis in cervical cancer by targeting STAT3. Oncotarget. 2015;6(28):25266

10. Hu Y, Liu C-M, Qi L, He T-Z, Shi-Guo L, Hao C-J, et al. Two common SNPs in pri-miR-125a alter the mature miRNA expression and associate with recurrent pregnancy loss in a Han-Chinese population. RNA Biol. 2011;8(5):861-72.

11. Stamatopoulos B, Meuleman N, Haibe-Kains B, Saussoy P, Van Den Neste E, Michaux $L$, et al. microRNA-29c and microRNA-223 down-regulation has in vivo significance in chronic lymphocytic leukemia and improves disease risk stratification. Blood. 2009:113(21):5237-45.

12. Chen $Q$, Wang H, Liu Y, Song Y, Lai L, Han Q, et al. Inducible microRNA-223 down-regulation promotes TLR-triggered IL-6 and IL-1 $\beta$ production in macrophages by targeting STAT3. PLoS ONE. 2012;7(8):e42971.
13. Kramer MF Stem-loop RT-qPCR for miRNAs. Curr Protocols Mol Biol. 2011;95(1):15.0. 1-.0..

14. Rodríguez D, Bretones G, Arango JR, Valdespino V, Campo E, Quesada $\checkmark$, et al. Molecular pathogenesis of CLL and its evolution. Int J Hematol. 2015:101(3):219-28.

15. Hallek M, Cheson BD, Catovsky D, Caligaris-Cappio F, Dighiero G, Döhner $\mathrm{H}$, et al. iwCLL guidelines for diagnosis, indications for treatment, response assessment, and supportive management of CLL. Blood. 2018;131(25):2745-60.

16. Amirpour M, Ayatollahi H, Sheikhi M, Azarkerdar S, Shams SF. Evaluation of BAALC gene expression in normal cytogenetic acute myeloid leukemia patients in north-east of Iran. Med J Islamic Rep Iran. 2016;30:418.

17. Mraz M, Malinova K, Kotaskova J, Pavlova S, Tichy B, Malcikova J, et al. miR-34a, miR-29c and miR-17-5p are downregulated in CLL patients with TP53 abnormalities. Leukemia. 2009;23(6):1159.

18. Haybar H, Shahrabi S, Rezaeeyan H, Jodat H, Saki N. Strategies to inhibit arsenic trioxide-induced cardiotoxicity in acute promyelocytic leukemia. J Cell Physiol. 2019;234(9):14500-6.

19. Klein U, Lia M, Crespo M, Siegel R, Shen Q, Mo T, et al. The DLEU2/miR15a/16-1 cluster controls B cell proliferation and its deletion leads to chronic lymphocytic leukemia. Cancer Cell. 2010;17(1):28-40.

20. Haybar H, Jalali MT, Zayeri ZD. What genetics tells us about cardiovascular disease in diabetic patients? Cardiovasc Haematol Disord Drug Targets. 2018;18(2):147-52.

21. Balatti , Tomasello L, Rassenti LZ, Veneziano D, Nigita G, Wang H-Y, et al MiR-125a and MiR-34a expression predicts Richter syndrome in chronic lymphocytic leukemia patients. Blood. 2018;132(20):2179-82.

22. Ahmadvand M, Eskandari M, Khakpour G, Pashaiefar H, Manoochehrabadi S, Yaghmaie M, et al. Identification of MiR-125a as a novel plasma diagnostic biomarker for chronic lymphoblastic leukemia. Clin Lab. 2019. https://doi.org/10.7754/clin.lab.2018.180815.

23. Rigolin GM, Saccenti E, Rizzotto L, Ferracin M, Martinelli S, Formigaro L, et al. Genetic subclonal complexity and miR125a-5p down-regulatio identify a subset of patients with inferior outcome in low-ris CLL patients. Oncotarget. 2014;5(1):140

24. Rodríguez-Vicente AE, Quwaider D, Benito R, Misiewicz-Krzeminska I, Hernández-Sánchez M, de Coca AG, et al. MicroRNA-223 is a novel negative regulator of HSP90B1 in CLL. BMC cancer. 2015;15(1):238.

25. Zhou K, Yi S, Yu Z, Li Z, Wang Y, Zou D, et al. MicroRNA-223 expression is uniformly down-regulated in B cell lymphoproliferative disorders and is associated with poor survival in patients with chronic lymphocytic leukemia. Leukemia Lymphoma. 2012:53(6):1155-61.

26. Tong Z, Liu N, Lin L, Guo X, Yang D, Zhang Q. miR-125a-5p inhibits cell proliferation and induces apoptosis in colon cancer via targeting $B C L 2$, BCL2L12 and MCL1. Biomed Pharmacother. 2015;75:129-36.

27. Bozec A, Zangari J, Butori-Pepino M, Ilie M, Lalvee S, Juhel T, et al. MiR223-3p inhibits angiogenesis and promotes resistance to cetuximab in head and neck squamous cell carcinoma. Oncotarget. 2017;8(34):57174.

\section{Publisher's Note}

Springer Nature remains neutral with regard to jurisdictional claims in published maps and institutional affiliations.

Ready to submit your research? Choose BMC and benefit from

- fast, convenient online submission

- thorough peer review by experienced researchers in your field

- rapid publication on acceptance

- support for research data, including large and complex data types

- gold Open Access which fosters wider collaboration and increased citations

- maximum visibility for your research: over 100M website views per year

At BMC, research is always in progress.

Learn more biomedcentral.com/submissions 